\title{
A combinatorial proof of a formula for Betti numbers of a stacked polytope
}

\author{
Suyoung Choi* \\ Department of Mathematical Sciences \\ KAIST, Republic of Korea \\ choisy@kaist.ac.kr
}

(Current) Department of Mathematics

Osaka City University, Japan

choi@sci.osaka-cu.ac.jp

Submitted: Aug 8, 2009; Accepted: Dec 13, 2009; Published: Jan 5, 2010

Mathematics Subject Classifications: 05A15, 05E40, 05E45, 52B05

\author{
Jang Soo $\mathrm{Kim}^{\dagger}$ \\ Department of Mathematical Sciences \\ KAIST, Republic of Korea \\ jskim@kaist.ac.kr \\ (Current) LIAFA \\ University of Paris 7, France
}

\begin{abstract}
For a simplicial complex $\Delta$, the graded Betti number $\beta_{i, j}(\mathbf{k}[\Delta])$ of the StanleyReisner ring $\mathbf{k}[\Delta]$ over a field $\mathbf{k}$ has a combinatorial interpretation due to Hochster. Terai and Hibi showed that if $\Delta$ is the boundary complex of a $d$-dimensional stacked polytope with $n$ vertices for $d \geqslant 3$, then $\beta_{k-1, k}(\mathbf{k}[\Delta])=(k-1)\left(\begin{array}{c}n-d \\ k\end{array}\right)$. We prove this combinatorially.
\end{abstract}

\section{Introduction}

A simplicial complex $\Delta$ on a finite set $V$ is a collection of subsets of $V$ satisfying

1. if $v \in V$, then $\{v\} \in \Delta$,

2. if $F \in \Delta$ and $F^{\prime} \subset F$, then $F^{\prime} \in \Delta$.

Each element $F \in \Delta$ is called a face of $\Delta$. The dimension of $F$ is defined by $\operatorname{dim}(F)=$ $|F|-1$. The dimension of $\Delta$ is defined by $\operatorname{dim}(\Delta)=\max \{\operatorname{dim}(F): F \in \Delta\}$. For a subset $W \subset V$, let $\Delta_{W}$ denote the simplicial complex $\{F \cap W: F \in \Delta\}$ on $W$.

*The research of the first author was carried out with the support of the Japanese Society for the Promotion of Science (JSPS grant no. P09023) and the Brain Korea 21 Project, KAIST.

${ }^{\dagger}$ The second author was supported by the SRC program of Korea Science and Engineering Foundation (KOSEF) grant funded by the Korea government (MEST) (No. R11-2007-035-01002-0). 
Let $\Delta$ be a simplicial complex on $V$. Two elements $v, u \in V$ are said to be connected if there is a sequence of vertices $v=u_{0}, u_{1}, \ldots, u_{r}=u$ such that $\left\{u_{i}, u_{i+1}\right\} \in \Delta$ for all $i=0,1, \ldots, r-1$. A connected component $C$ of $\Delta$ is a maximal nonempty subset of $V$ such that every two elements of $C$ are connected.

Let $V=\left\{x_{1}, x_{2}, \ldots, x_{n}\right\}$ and let $R$ be the polynomial ring $\mathbf{k}\left[x_{1}, \ldots, x_{n}\right]$ over a fixed field $\mathbf{k}$. Then $R$ is a graded ring with the standard grading $R=\oplus_{i \geqslant 0} R_{i}$. Let $R(-j)=$ $\oplus_{i \geqslant 0}(R(-j))_{i}$ be the graded module over $R$ with $(R(-j))_{i}=R_{j+i}$. The Stanley-Reisner ring $\mathbf{k}[\Delta]$ of $\Delta$ over $\mathbf{k}$ is defined to be $R / I_{\Delta}$, where $I_{\Delta}$ is the ideal of $R$ generated by the monomials $x_{i_{1}} x_{i_{2}} \cdots x_{i_{r}}$ such that $\left\{x_{i_{1}}, x_{i_{2}}, \ldots, x_{i_{r}}\right\} \notin \Delta$. A finite free resolution of $\mathbf{k}[\Delta]$ is an exact sequence

$$
0 \longrightarrow F_{r} \stackrel{\phi_{r}}{\longrightarrow} F_{r-1} \stackrel{\phi_{r-1}}{\longrightarrow} \cdots \stackrel{\phi_{2}}{\longrightarrow} F_{1} \stackrel{\phi_{1}}{\longrightarrow} F_{0} \stackrel{\phi_{0}}{\longrightarrow} \mathbf{k}[\Delta] \longrightarrow 0
$$

where $F_{i}=\oplus_{j \geqslant 0} R(-j)^{\beta_{i, j}}$ and each $\phi_{i}$ is degree-preserving. A finite free resolution (11) is minimal if each $\beta_{i, j}$ is smallest possible. There is a minimal finite free resolution of $\mathbf{k}[\Delta]$ and it is unique up to isomorphism. If (1D) is minimal, then the $(i, j)$-th graded Betti number $\beta_{i, j}(\mathbf{k}[\Delta])$ of $\mathbf{k}[\Delta]$ is defined to be $\beta_{i, j}(\mathbf{k}[\Delta])=\beta_{i, j}$. Hochster's theorem says

$$
\beta_{i, j}(\mathbf{k}[\Delta])=\sum_{\substack{W \subset V \\|W|=j}} \operatorname{dim}_{\mathbf{k}} \widetilde{H}_{j-i-1}\left(\Delta_{W} ; \mathbf{k}\right) .
$$

We refer the reader to [1, 5] for the details of Betti numbers and Hochster's theorem. Since $\operatorname{dim}_{\mathbf{k}} \widetilde{H}_{0}\left(\Delta_{W} ; \mathbf{k}\right)$ is the number of connected components of $\Delta_{W}$ minus 1 , we can interpret $\beta_{i-1, i}(\mathbf{k}[\Delta])$ in a purely combinatorial way.

Definition 1.1. Let $\Delta$ be a simplicial complex on a finite nonempty set $V$. Let $k$ be a nonnegative integer. The $k$-th special graded Betti number $b_{k}(\Delta)$ of $\Delta$ is defined to be

$$
b_{k}(\Delta)=\sum_{\substack{W \subset V \\|W|=k}}\left(\operatorname{cc}\left(\Delta_{W}\right)-1\right)
$$

where $\operatorname{cc}\left(\Delta_{W}\right)$ denotes the number of connected components of $\Delta_{W}$.

Note that since there is no connected component in $\Delta_{\emptyset}=\{\emptyset\}$, we have $b_{0}(\Delta)=-1$. If $k>|V|$, then $b_{k}(\Delta)=0$ because there is nothing in the sum in (2). Thus we have

$$
b_{k}(\Delta)= \begin{cases}\beta_{k-1, k}(\mathbf{k}[\Delta]), & \text { if } k \geqslant 1, \\ -1, & \text { if } k=0\end{cases}
$$

We refer the reader to [7] for the basic notions of convex polytopes. Let $P$ be a simplicial polytope with vertex set $V$. The boundary complex $\Delta(P)$ is the simplicial complex $\Delta$ on $V$ such that $F \in \Delta$ for some $F \subset V$ if and only if $F \neq V$ and the convex hull of $F$ is a face of $P$. Note that if the dimension of $P$ is $d$, then $\operatorname{dim}(\Delta(P))=d-1$. 
For a $d$-dimensional simplicial polytope $P$, we can attach a $d$-dimensional simplex to a facet of $P$. A stacked polytope is a simplicial polytope obtained in this way starting with a $d$-dimensional simplex.

Let $P$ be a $d$-dimensional stacked polytope with $n$ vertices. Hibi and Terai [ $[6]$ showed that $\beta_{i, j}(\mathbf{k}[\Delta(P)])=0$ unless $i=j-1$ or $i=j-d+1$. Since $\beta_{i-1, i}(\mathbf{k}[\Delta(P)])=$ $\beta_{n-i-d+1, n-i}(\mathbf{k}[\Delta(P)])$, it is sufficient to determine $\beta_{i-1, i}(\mathbf{k}[\Delta(P)])$ to find all $\beta_{i, j}(\mathbf{k}[\Delta(P)])$. In the same paper, they found the following formula for $\beta_{k-1, k}(\mathbf{k}[\Delta(P)])$ :

$$
\beta_{k-1, k}(\mathbf{k}[\Delta(P)])=(k-1)\left(\begin{array}{c}
n-d \\
k
\end{array}\right) .
$$

Herzog and Li Marzi [4 gave another proof of (3).

The main purpose of this paper is to prove (3) combinatorially. In the meanwhile, we get as corollaries the results of Bruns and Hibi 2] : a formula of $b_{k}(\Delta)$ if $\Delta$ is a tree (or a cycle) considered as a 1-dimensional simplicial complex.

\section{Definition of $t$-connected sum}

In this section we define a $t$-connected sum of simplicial complexes, which gives another equivalent definition of the boundary complex of a stacked polytope. See [3] for the details of connected sums. And then, we extend the definition of $t$-connected sum to graphs, which has less restrictions on the construction. Every graph in this paper is simple.

\subsection{A $t$-connected sum of simplicial complexes}

Let $V$ and $V^{\prime}$ be finite sets. A relabeling is a bijection $\sigma: V \rightarrow V^{\prime}$. If $\Delta$ is a simplicial complex on $V$, then $\sigma(\Delta)=\{\sigma(F): F \in \Delta\}$ is a simplicial complex on $V^{\prime}$.

Definition 2.1. Let $\Delta_{1}$ and $\Delta_{2}$ be simplicial complexes on $V_{1}$ and $V_{2}$ respectively. Let $F_{1} \in \Delta_{1}$ and $F_{2} \in \Delta_{2}$ be maximal faces with $\left|F_{1}\right|=\left|F_{2}\right|$. Let $V_{2}^{\prime}$ be a finite set and $\sigma: V_{2} \rightarrow V_{2}^{\prime}$ a relabeling such that $V_{1} \cap V_{2}^{\prime}=F_{1}$ and $\sigma\left(F_{2}\right)=F_{1}$. Then the connected sum $\Delta_{1} \#_{\sigma}^{F_{1}, F_{2}} \Delta_{2}$ of $\Delta_{1}$ and $\Delta_{2}$ with respect to $\left(F_{1}, F_{2}, \sigma\right)$ is the simplicial complex $\left(\Delta_{1} \cup\right.$ $\left.\sigma\left(\Delta_{2}\right)\right) \backslash\left\{F_{1}\right\}$ on $V_{1} \cup V_{2}^{\prime}$. If $\Delta=\Delta_{1} \#_{\sigma}^{F_{1}, F_{2}} \Delta_{2}$ and $\left|F_{1}\right|=\left|F_{2}\right|=t$, then we say that $\Delta$ is a $t$-connected sum of $\Delta_{1}$ and $\Delta_{2}$.

Note that if $\Delta_{1}$ and $\Delta_{2}$ are $(d-1)$-dimensional pure simplicial complexes, i.e., the dimension of each maximal face is $d-1$, then we can only define a $d$-connected sum of them.

Let $\Delta_{1}, \Delta_{2}, \ldots, \Delta_{n}$ be simplicial complexes. A simplicial complex $\Delta$ is said to be a $t$ connected sum of $\Delta_{1}, \ldots, \Delta_{n}$ if there is a sequence of simplicial complexes $\Delta_{1}^{\prime}, \Delta_{2}^{\prime}, \ldots, \Delta_{n}^{\prime}$ such that $\Delta_{1}^{\prime}=\Delta_{1}, \Delta_{i}^{\prime}$ is a $t$-connected sum of $\Delta_{i-1}^{\prime}$ and $\Delta_{i}$ for $i=2,3, \ldots, n$, and $\Delta_{n}^{\prime}=\Delta$. 


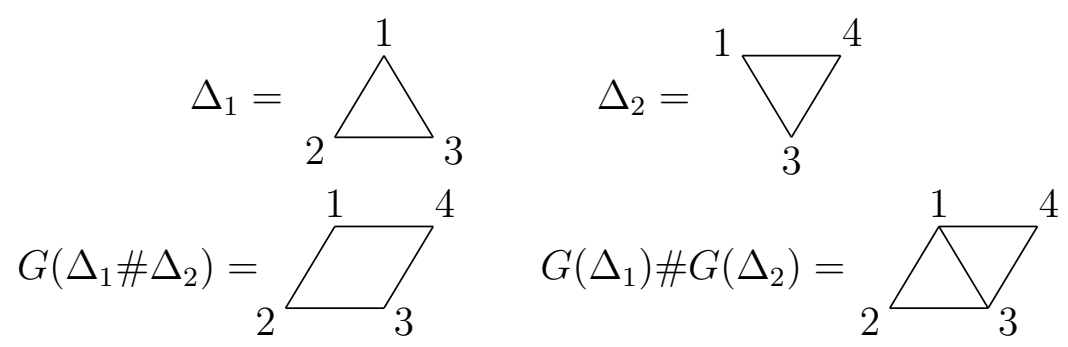

Figure 1: The 1-skeleton of a 2-connected sum of $\Delta_{1}$ and $\Delta_{2}$ is not a 2-connected sum of $G\left(\Delta_{1}\right)$ and $G\left(\Delta_{2}\right)$.

\subsection{A $t$-connected sum of graphs}

Let $G$ be a graph with vertex set $V$ and edge set $E$. Let $W \subset V$. Then the induced subgraph $\left.G\right|_{W}$ of $G$ with respect to $W$ is the graph with vertex set $W$ and edge set $\{\{x, y\} \in E: x, y \in W\}$. Let

$$
b_{k}(G)=\sum_{\substack{W \subset V \\|W|=k}}\left(\operatorname{cc}\left(\left.G\right|_{W}\right)-1\right),
$$

where $\operatorname{cc}\left(\left.G\right|_{W}\right)$ denotes the number of connected components of $\left.G\right|_{W}$.

Let $\Delta$ be a simplicial complex on $V$. The 1 -skeleton $G(\Delta)$ of $\Delta$ is the graph with vertex set $V$ and edge set $E=\{F \in \Delta:|F|=2\}$. By definition, the connected components of $\Delta_{W}$ and $\left.G(\Delta)\right|_{W}$ are identical for all $W \subset V$. Thus $b_{k}(\Delta)=b_{k}(G(\Delta))$.

Now we define a $t$-connected sum of two graphs.

Definition 2.2. Let $G_{1}$ and $G_{2}$ be graphs with vertex sets $V_{1}$ and $V_{2}$, and edge sets $E_{1}$ and $E_{2}$ respectively. Let $F_{1} \subset V_{1}$ and $F_{2} \subset V_{2}$ be sets of vertices such that $\left|F_{1}\right|=\left|F_{2}\right|$, and $\left.G_{1}\right|_{F_{1}}$ and $\left.G_{2}\right|_{F_{2}}$ are complete graphs. Let $V_{2}^{\prime}$ be a finite set and $\sigma: V_{2} \rightarrow V_{2}^{\prime}$ a relabeling such that $V_{1} \cap V_{2}^{\prime}=F_{1}$ and $\sigma\left(F_{2}\right)=F_{1}$. Then the connected sum $G_{1} \#_{\sigma}^{F_{1}, F_{2}} G_{2}$ of $G_{1}$ and $G_{2}$ with respect to $\left(F_{1}, F_{2}, \sigma\right)$ is the graph with vertex set $V_{1} \cup V_{2}^{\prime}$ and edge set $E_{1} \cup \sigma\left(E_{2}\right)$, where $\sigma\left(E_{2}\right)=\left\{\{\sigma(x), \sigma(y)\}:\{x, y\} \in E_{2}\right\}$. If $G=G_{1} \#_{\sigma}^{F_{1}, F_{2}} G_{2}$ and $\left|F_{1}\right|=\left|F_{2}\right|=t$, then we say that $G$ is a $t$-connected sum of $G_{1}$ and $G_{2}$.

Note that in contrary to the definition of $t$-connected sum of simplicial complexes, it is not required that $F_{1}$ and $F_{2}$ are maximal, and we do not remove any element in $E_{1} \cup \sigma\left(E_{2}\right)$. We define a $t$-connected sum of $G_{1}, G_{2}, \ldots, G_{n}$ as we did for simplicial complexes.

It is easy to see that, if $\left|F_{1}\right|=\left|F_{2}\right| \geqslant 3$, then $G\left(\Delta_{1} \#_{\sigma}^{F_{1}}, F_{2} \Delta_{2}\right)=G\left(\Delta_{1}\right) \#_{\sigma}^{F_{1}, F_{2}} G\left(\Delta_{2}\right)$. Thus we get the following proposition.

Proposition 2.3. For $t \geqslant 3$, if $\Delta$ is a t-connected sum of $\Delta_{1}, \Delta_{2}, \ldots, \Delta_{n}$, then $G(\Delta)$ is a t-connected sum of $G\left(\Delta_{1}\right), G\left(\Delta_{2}\right), \ldots, G\left(\Delta_{n}\right)$.

Note that Proposition 2.3 is not true if $t=2$ as the following example shows.

Example 2.4. Let $\Delta_{1}=\{12,23,13\}$ and $\Delta_{2}=\{13,34,14\}$ be simplicial complexes on $V_{1}=\{1,2,3\}$ and $V_{2}=\{1,3,4\}$. Here 12 means the set $\{1,2\}$. Let $F_{1}=F_{2}=\{1,3\}$ and let $\sigma$ be the identity map from $V_{2}$ to itself. Then the edge set of $G\left(\Delta_{1} \#_{\sigma}^{F_{1}, F_{2}} \Delta_{2}\right)$ is $\{12,23,34,14\}$, but the edge set of $G\left(\Delta_{1}\right) \#_{\sigma}^{F_{1}, F_{2}} G\left(\Delta_{2}\right)$ is $\{12,23,34,14,13\}$. See Figure 1. 


\section{Main results}

In this section we find a formula of $b_{k}(G)$ for a graph $G$ which is a $t$-connected sum of two graphs. To do this let us introduce the following notation. For a graph $G$ with vertex set $V$, let

$$
c_{k}(G)=\sum_{\substack{W \subset V \\|W|=k}} \operatorname{cc}\left(\left.G\right|_{W}\right) .
$$

Note that $c_{k}(G)=b_{k}(G)+\left(\begin{array}{c}|V| \\ k\end{array}\right)$.

Lemma 3.1. Let $G_{1}$ and $G_{2}$ be graphs with $n_{1}$ and $n_{2}$ vertices respectively. Let $t$ be a positive integer and let $G$ be a t-connected sum of $G_{1}$ and $G_{2}$. Then

$$
\begin{aligned}
c_{k}(G)= & \sum_{i=0}^{k}\left(c_{i}\left(G_{1}\right)\left(\begin{array}{c}
n_{2}-t \\
k-i
\end{array}\right)+c_{i}\left(G_{2}\right)\left(\begin{array}{c}
n_{1}-t \\
k-i
\end{array}\right)\right) \\
& -\left(\begin{array}{c}
n_{1}+n_{2}-t \\
k
\end{array}\right)+\left(\begin{array}{c}
n_{1}+n_{2}-2 t \\
k
\end{array}\right) .
\end{aligned}
$$

Proof. Let $V_{1}$ (resp. $V_{2}$ ) be the vertex set of $G_{1}$ (resp. $G_{2}$ ). We have $G=G_{1} \#_{\sigma}^{F_{1}, F_{2}} G_{2}$ for some $F_{1} \subset V_{1}, F_{2} \subset V_{2}$, a vertex set $V_{2}^{\prime}$ and a relabeling $\sigma: V_{1} \rightarrow V_{2}^{\prime}$ such that $V_{1} \cap V_{2}^{\prime}=F_{1}, \sigma\left(F_{2}\right)=F_{1}$, and $\left.G_{1}\right|_{F_{1}}$ and $\left.G_{2}\right|_{F_{2}}$ are complete graphs on $t$ vertices.

Let $A$ be the set of pairs $(C, W)$ such that $W \subset V_{1} \cup V_{2}^{\prime},|W|=k$ and $C$ is a connected component of $\left.G\right|_{W}$. Let

$$
A_{1}=\left\{(C, W) \in A: C \cap V_{1} \neq \emptyset\right\}, \quad A_{2}=\left\{(C, W) \in A: C \cap V_{2}^{\prime} \neq \emptyset\right\} .
$$

Then $c_{k}(G)=|A|=\left|A_{1}\right|+\left|A_{2}\right|-\left|A_{1} \cap A_{2}\right|$. It is sufficient to show that $\left|A_{1}\right|=$ $\sum_{i=0}^{k} c_{i}\left(G_{1}\right)\left(\begin{array}{c}n_{2}-t \\ k-i\end{array}\right),\left|A_{2}\right|=\sum_{i=0}^{k} c_{i}\left(G_{2}\right)\left(\begin{array}{c}n_{1}-t \\ k-i\end{array}\right)$ and $\left|A_{1} \cap A_{2}\right|=\left(\begin{array}{c}n_{1}+n_{2}-t \\ k\end{array}\right)-\left(\begin{array}{c}n_{1}+n_{2}-2 t \\ k\end{array}\right)$.

Let $B_{1}$ be the set of triples $\left(C_{1}, W_{1}, X\right)$ such that $W_{1} \subset V_{1}, X \subset V_{2}^{\prime} \backslash V_{1},|X|+\left|W_{1}\right|=k$ and $C_{1}$ is a connected component of $\left.G_{1}\right|_{W_{1}}$. Let $\phi_{1}: A_{1} \rightarrow B_{1}$ be the map defined by $\phi_{1}(C, W)=\left(C \cap V_{1}, W \cap V_{1}, W \backslash V_{1}\right)$. Then $\phi_{1}$ has the inverse map defined as follows. For a triple $\left(C_{1}, W_{1}, X\right) \in B_{1}, \phi_{1}^{-1}\left(C_{1}, W_{1}, X\right)=(C, W)$, where $W=W_{1} \cup X$ and $C$ is the connected component of $\left.G\right|_{W}$ containing $C_{1}$. Thus $\phi_{1}$ is a bijection and we get $\left|A_{1}\right|=\left|B_{1}\right|=\sum_{i=0}^{k} c_{i}\left(G_{1}\right)\left(\begin{array}{c}n_{2}-t \\ k-i\end{array}\right)$. Similarly we get $\left|A_{2}\right|=\sum_{i=0}^{k} c_{i}\left(G_{2}\right)\left(\begin{array}{c}n_{1}-t \\ k-i\end{array}\right)$.

Now let $B=\left\{W \subset V_{1} \cup V_{2}^{\prime}: W \cap F_{1} \neq \emptyset\right\}$. Let $\psi: A_{1} \cap A_{2} \rightarrow B$ be the map defined by $\psi(C, W)=W$. We have the inverse map $\psi^{-1}$ as follows. For $W \in B, \psi^{-1}(W)=(C, W)$, where $C$ is the connected component of $\left.G\right|_{W}$ containing $W \cap F_{1}$, which is guaranteed to exist since $\left.G\right|_{F_{1}}=\left.G_{1}\right|_{F_{1}}$ is a complete graph. Thus $\psi$ is a bijection, and we get $\left|A_{1} \cap A_{2}\right|=|B|=\left(\begin{array}{c}n_{1}+n_{2}-t \\ k\end{array}\right)-\left(\begin{array}{c}n_{1}+n_{2}-2 t \\ k\end{array}\right)$.

Theorem 3.2. Let $G_{1}$ and $G_{2}$ be graphs with $n_{1}$ and $n_{2}$ vertices respectively. Let $t$ be a positive integer and let $G$ be a t-connected sum of $G_{1}$ and $G_{2}$. Then

$$
b_{k}(G)=\sum_{i=0}^{k}\left(b_{i}\left(G_{1}\right)\left(\begin{array}{c}
n_{2}-t \\
k-i
\end{array}\right)+b_{i}\left(G_{2}\right)\left(\begin{array}{c}
n_{1}-t \\
k-i
\end{array}\right)\right)+\left(\begin{array}{c}
n_{1}+n_{2}-2 t \\
k
\end{array}\right) .
$$


Proof. Since $c_{k}(G)=b_{k}(G)+\left(\begin{array}{c}n_{1}+n_{2}-t \\ k\end{array}\right), c_{i}\left(G_{1}\right)=b_{i}\left(G_{1}\right)+\left(\begin{array}{c}n_{1} \\ i\end{array}\right)$ and $c_{i}\left(G_{2}\right)=b_{i}\left(G_{2}\right)+\left(\begin{array}{c}n_{2} \\ i\end{array}\right)$, by Lemma 3.1, it is sufficient to show that

$$
2\left(\begin{array}{c}
n_{1}+n_{2}-t \\
k
\end{array}\right)=\sum_{i=0}^{k}\left(\left(\begin{array}{c}
n_{1} \\
i
\end{array}\right)\left(\begin{array}{c}
n_{2}-t \\
k-i
\end{array}\right)+\left(\begin{array}{c}
n_{2} \\
i
\end{array}\right)\left(\begin{array}{c}
n_{1}-t \\
k-i
\end{array}\right)\right)
$$

which is immediate from the identity $\sum_{i=0}^{k}\left(\begin{array}{c}a \\ i\end{array}\right)\left(\begin{array}{c}b \\ k-i\end{array}\right)=\left(\begin{array}{c}a+b \\ k\end{array}\right)$.

Recall that a $t$-connected sum $G$ of two graphs depends on the choice of vertices of each graph and the identification of the chosen vertices. However, Theorem 3.2 says that $b_{k}(G)$ does not depend on them. Thus we get the following important property of a $t$-connected sum of graphs.

Corollary 3.3. Let $t$ be a positive integer and let $G$ be a $t$-connected sum of graphs $G_{1}, G_{2}, \ldots, G_{n}$. If $H$ is also a $t$-connected sum of $G_{1}, G_{2}, \ldots, G_{n}$, then $b_{k}(G)=b_{k}(H)$ for all $k$.

Using Proposition 2.3, we get a formula for the special graded Betti number of a $t$-connected sum of two simplicial complexes for $t \geqslant 3$.

Corollary 3.4. Let $\Delta_{1}$ and $\Delta_{2}$ be simplicial complexes on $V_{1}$ and $V_{2}$ respectively with $\left|V_{1}\right|=n_{1}$ and $\left|V_{2}\right|=n_{2}$. Let $t$ be a positive integer and let $\Delta$ be a $t$-connected sum of $\Delta_{1}$ and $\Delta_{2}$. If $t \geqslant 3$, then

$$
b_{k}(\Delta)=\sum_{i=0}^{k}\left(b_{i}\left(\Delta_{1}\right)\left(\begin{array}{c}
n_{2}-t \\
k-i
\end{array}\right)+b_{i}\left(\Delta_{2}\right)\left(\begin{array}{c}
n_{1}-t \\
k-i
\end{array}\right)\right)+\left(\begin{array}{c}
n_{1}+n_{2}-2 t \\
k
\end{array}\right) .
$$

For an integer $n$, let $K_{n}$ denote a complete graph with $n$ vertices.

Let $G$ be a graph with vertex set $V$. If $H$ is a $t$-connected sum of $G$ and $K_{t+1}$, then $H$ is a graph obtained from $G$ by adding a new vertex $v$ connected to all vertices in $W$ for some $W \subset V$ such that $\left.G\right|_{W}$ is isomorphic to $K_{t}$. Thus $H$ is determined by choosing such a subset $W \subset V$. Using this observation, we get the following lemma.

Theorem 3.5. Let $t$ be a positive integer. Let $G$ be a t-connected sum of $n K_{t+1}$ 's. Then

$$
b_{k}(G)=(k-1)\left(\begin{array}{l}
n \\
k
\end{array}\right) \text {. }
$$

Proof. We construct a sequence of graphs $H_{1}, \ldots, H_{n}$ as follows. Let $H_{1}$ be the complete graph with vertex set $\left\{v_{1}, v_{2}, \ldots, v_{t+1}\right\}$. For $i \geqslant 2$, let $H_{i}$ be the graph obtained from $H_{i-1}$ by adding a new vertex $v_{t+i}$ connected to all vertices in $\left\{v_{1}, v_{2}, \ldots, v_{t}\right\}$. Then $H_{n}$ is a $t$-connected sum of $n K_{t+1}$ 's, and we have $b_{k}(G)=b_{k}\left(H_{n}\right)$ by Corollary 3.3. In $H_{n}$, the vertex $v_{i}$ is connected to all the other vertices for $i \leqslant t$, and $v_{j}$ and $v_{j^{\prime}}$ are not connected to each other for all $t+1 \leqslant j, j^{\prime} \leqslant t+n$. Thus $b_{k}\left(H_{n}\right)=(k-1)\left(\begin{array}{l}n \\ k\end{array}\right)$.

Observe that every tree with $n+1$ vertices is a 1 -connected sum of $n K_{2}$ 's. Thus we get the following nontrivial property of trees which was observed by Bruns and Hibi [2]. 
Corollary 3.6. [2, Example 2.1. (b)] Let $T$ be a tree with $n+1$ vertices. Then $b_{k}(T)$ does not depend on the specific tree $T$. We have

$$
b_{k}(T)=(k-1)\left(\begin{array}{l}
n \\
k
\end{array}\right) .
$$

Corollary 3.7. [2, Example 2.1. (c)] Let $G$ be an $n$-gon. If $k=n$, then $b_{k}(G)=0$; otherwise,

$$
b_{k}(G)=\frac{n(k-1)}{n-k}\left(\begin{array}{c}
n-2 \\
k
\end{array}\right) .
$$

Proof. It is clear for $k=n$. Assume $k<n$. Let $V=\left\{v_{1}, \ldots, v_{n}\right\}$ be the vertex set of $G$. Then

$$
\begin{aligned}
(n-k) \cdot b_{k}(G) & =\sum_{\substack{W \subset V \\
|W|=k}}\left(\operatorname{cc}\left(\left.G\right|_{W}\right)-1\right) \sum_{v \in V \backslash W} 1 \\
& =\sum_{v \in V} \sum_{\substack{W \subset V \backslash\{v\} \\
|W|=k}}\left(\operatorname{cc}\left(\left.G\right|_{W}\right)-1\right) \\
& =\sum_{v \in V} b_{k}\left(\left.G\right|_{V \backslash\{v\}}\right) .
\end{aligned}
$$

Since each $\left.G\right|_{V \backslash\{v\}}$ is a tree with $n-1$ vertices, we are done by Corollary 3.6.

Remark 3.8. Bruns and Hibi [2] obtained Corollary 3.6 and Corollary 3.7 by showing that if $\Delta$ is a tree (or an $n$-gon), considered as a 1-dimensional simplicial complex, then $\mathbf{k}[\Delta]$ has a pure resolution. Since $\mathbf{k}[\Delta]$ is Cohen-Macaulay and it has a pure resolution, the Betti numbers are determined by its type (c.f. [1).

Now we can prove (3). Note that, for $d \geqslant 3$, if $P$ is a $d$-dimensional simplicial polytope and $Q$ is a simplicial polytope obtained from $P$ by attaching a $d$-dimensional simplex $S$ to a facet of $P$, then $\Delta(Q)$ is a $d$-connected sum of $\Delta(P)$ and $\Delta(S)$, and thus the 1skeleton $G(\Delta(Q))$ is a $d$-connected sum of $G(\Delta(P))$ and $K_{d+1}$. Hence the 1-skeleton of the boundary complex of a $d$-dimensional stacked polytope is a $d$-connected sum of $K_{d+1}$ 's.

Theorem 3.9. Let $P$ be a d-dimensional stacked polytope with $n$ vertices. If $d \geqslant 3$, then

$$
b_{k}(\Delta(P))=(k-1)\left(\begin{array}{c}
n-d \\
k
\end{array}\right) .
$$

If $d=2$, then

$$
b_{k}(\Delta(P))= \begin{cases}0, & \text { if } k=n, \\
\frac{n(k-1)}{n-k}\left(\begin{array}{c}
n-2 \\
k
\end{array}\right), & \text { otherwise. }\end{cases}
$$

Proof. Assume $d \geqslant 3$. Then the 1 -skeleton $G(\Delta(P))$ is a $d$-connected sum of $n-d K_{d+1}$ 's. Thus by Theorem 3.5, we get $b_{k}(\Delta(P))=b_{k}(G(\Delta(P)))=(k-1)\left(\begin{array}{c}n-d \\ k\end{array}\right)$.

Now assume $d=2$. Then $G(\Delta(P))$ is an $n$-gon. Thus by Corollary 3.7 we are done. 


\section{References}

[1] Winfried Bruns and Jürgen Herzog. Cohen-Macaulay rings, volume 39 of Cambridge Studies in Advanced Mathematics. Cambridge University Press, Cambridge, 1993.

[2] Winfried Bruns and Takayuki Hibi. Cohen-Macaulay partially ordered sets with pure resolutions. European J. Combin., 19(7):779-785, 1998.

[3] Victor M. Buchstaber and Taras E. Panov. Torus actions and their applications in topology and combinatorics, volume 24 of University Lecture Series. American Mathematical Society, Providence, RI, 2002.

[4] Jürgen Herzog and Enzo Maria Li Marzi. Bounds for the Betti numbers of shellable simplicial complexes and polytopes. In Commutative algebra and algebraic geometry (Ferrara), volume 206 of Lecture Notes in Pure and Appl. Math., pages 157-167. Dekker, New York, 1999.

[5] Richard P. Stanley. Combinatorics and commutative algebra, volume 41 of Progress in Mathematics. Birkhäuser Boston Inc., Boston, MA, second edition, 1996.

[6] Naoki Terai and Takayuki Hibi. Computation of Betti numbers of monomial ideals associated with stacked polytopes. Manuscripta Math., 92(4):447-453, 1997.

[7] Günter M. Ziegler. Lectures on polytopes, volume 152 of Graduate Texts in Mathematics. Springer-Verlag, New York, 1995. 